\title{
EQUAÇÕES ALOMÉTRICAS PARA ESTIMATIVA DE CARBONO EM ÁRVORES DE UMA ÁREA URBANA EM VIÇOSA-MG ${ }^{1}$
}

Daniel Brianezi², Laércio Antônio Gonçalves Jacovine³ ${ }^{3}$ Carlos Pedro Boechat Soares ${ }^{3}$, Renato Vinícius Oliveira Castro ${ }^{4}$ e Vanessa Maria Basso ${ }^{2}$

\begin{abstract}
RESUMO - Objetivou-se com este estudo gerar equações para estimar o carbono presente na arborização do campus-sede da Universidade Federal de Viçosa, contribuindo para o conhecimento sobre a capacidade das áreas verdes urbanas no sequestro e estocagem de carbono. Assim, inventariaram-se todas as árvores com DAP igual ou superior a $5 \mathrm{~cm}$ presentes na Universidade. Para as árvores não palmeiras, selecionaram-se 721 árvores-amostra, que foram cubadas rigorosamente em pé através da aplicação sucessiva da expressão de Smalian até o diâmetro-limite de $5 \mathrm{~cm}$, tanto para o fuste quanto para os galhos. No caso das palmeiras, cubaram-se $100 \%$ dos indivíduos utilizando a expressão de Huber. A densidade básica da madeira com casca e o teor de carbono foram obtidos, nas palmeiras, com a retirada de um disco na porção do DAP, dada a dificuldade de tradagem. Já nas demais árvores, utilizou-se um trado mecânico. Com base no volume, na densidade básica e no teor de carbono, calcularam-se o carbono total e o carbono dos galhos. Ademais, avaliaram-se os modelos de Schumacher e Hall (1933) e Spurr (1952), modificado para estimar o carbono fixado nesses indivíduos. Nas árvores não palmeiras do campus-sede da Universidade Federal de Viçosa, o carbono total e o carbono dos galhos podem ser estimados, em kg, em razão do Diâmetro à Altura do Peito (DAP, em cm) e da Altura Total (Ht, em m), por -0,906586+1,60421*LnDAP+0,37162*LnHt e por -2,052673+1,89903*LnDAP+0,24156*LnHt, respectivamente. Nas palmeiras, o carbono total pode ser estimado por -4,46988+199082*LnDAP+1,06420*LnHt.
\end{abstract}

Palavras-chave: Arborização; Estoque de carbono; Modelagem.

\section{ALLOMETRIC EQUATIONS FOR ESTIMATING CARBON OF URBAN TREES IN VIÇOSA-MG}

\begin{abstract}
The objective of this study was to generate equations to estimate carbon in trees of the main campus of Universidade Federal de Viçosa, contributing to the knowledge about the ability of urban green areas in removing and storing carbon. Thus, every tree with DBH less than $5 \mathrm{~cm}$ located at the university was inventoried. For no palm trees, were selected 721 sample trees, which were rigorously cubed by successive application of Smalian expression until $5 \mathrm{~cm}$ of diameter, for trunk and twigs. Regarding the palm trees, $100 \%$ of the individuals were cubed using the Huber expression. The basic density of wood with bark and content carbon were obtained, in palm trees, by removing a disk on DBH, due to the difficult of borehole. For the other trees, a mechanical auger was used. Based on the volume with bark, the basic density and carbon content, we calculated the total carbon and carbon in the twigs. Furthermore, we evaluated the Schumacher \& Hall (1933) and Spurr (1952) modified models to estimate the carbon fixed in these individuals. In the case of no palm trees, the total carbon and the branches carbon can be estimated, in kg, according to the Diameter at Breast Height (DBH in cm) and Total Height (TH, in meters), by-0,906586+1,60421*LnDAP+0,37162*LnHt e por $-2,052673+1,89903 * L n D A P+0,24156 * L n H t$, in this order. In palms, the total carbon can be estimated by $-4,46988+199082 * \operatorname{LnDBH}+1,06420 * \operatorname{LnTH}$.
\end{abstract}

Keyword: Afforestation; Carbon stock; Modeling.

\footnotetext{
${ }^{1}$ Recebido em 11.10.2012 aceito para publicação em 15.10.2013.

${ }^{2}$ Programa de Pós-graduação em Ciência Florestal, Universidade Federal de Viçosa, UFV, Brasil. Email: < daniel.floresta@yahoo.com.br> e <nessabasso@yahoo.com.br>.

${ }^{3}$ Departamento de Engenharia Florestal, Universidade Federal de Viçosa, UFV, Brasil. E-mail: < jacovine@ufv.br> e <csoares@ufv.br>.

${ }^{4}$ Universidade de Brasília, UNB, Brasil. E-mail:<castrorvo@ymail.com>.
} 


\section{INTRODUÇÃO}

Diante de um cenário de mudança no clima global, as florestas têm ganhado notoriedade pelo seu importante papel na remoção do $\mathrm{CO}_{2}$ atmosférico e na sua conversão em biomassa. As árvores têm função importante na minimização da concentração dos gases de efeito-estufa, pois captam e estocam o $\mathrm{CO}_{2}$ presente na atmosfera. Sabe-se que esse gás captado é convertido em biomassa durante o crescimento da árvore. Dessa forma, as árvores retêm o carbono nos seus constituintes, sendo esse o elemento mais abundante na biomassa. Entretanto, existe grande variação na quantidade de carbono estocado pelas diferentes formações e espécies florestais.

Vários trabalhos já vêm sendo desenvolvidos em florestas nativas e plantadas com o objetivo de conhecer o potencial dessas áreas na estocagem de carbono, como os desenvolvidos por Soares e Oliveira (2002) e Soares et al. (2005) para plantios de eucalipto, por Urbano et al. (2008) para estimar o carbono em árvores de bracatinga e por Scolforo et al. (2008), que compilaram equações para estimar o carbono em diferentes fisionomias da flora nativa mineira. Entretanto, são limitados os trabalhos referentes à arborização urbana, o que resulta no desconhecimento sobre o potencial de fixação de carbono pelas árvores presentes nas cidades, seja pela dificuldade de obtenção de dados sobre volume e biomassa ou, mesmo, pelo quadro de desinteresse por tais áreas.

As árvores urbanas, além dos diversos benefícios já conhecidos pela sociedade, podem apresentar contribuição muito significativa à remoção de $\mathrm{CO}_{2}$, se houver espaço, manutenção, boas condições de iluminação e umidade, além de boas condições de fertilidade para as espécies (SAMPSON et al., 1992), especialmente pelo fato de poderem estocar carbono por mais tempo, em comparação com as florestas comerciais em razão, justamente, dos objetivos do seu plantio.

Para estimar a biomassa e quantificar o estoque de carbono orgânico em árvores, há métodos que vão desde a análise destrutiva com o abate das árvores (métodos diretos) e não destrutiva (métodos indiretos) até a estimativa por processamento digital de imagens por satélite (GATTO et al., 2011).

O uso de modelos alométricos para estimar o estoque de carbono em florestas é prática mais comum, principalmente, em reflorestamentos com espécies comerciais (SOARES; OLIVEIRA, 2002; PAIXÃO et al., 2006; GATTO et al., 2011). Um modelo alométrico é uma relação empírica entre a variável de interesse (neste caso, o estoque de carbono) e as variáveis facilmente mensuráveis, a exemplo da altura da árvore e diâmetro da árvore à altura do peito (DAP), que podem ser estabelecidas por meio de uma análise de regressão (KETTERINGS et al., 2001; RIBEIRO et al., 2011).

Nesse sentido, objetivou-se com este estudo gerar equações para estimar o carbono presente em uma área arborizada, contribuindo para o conhecimento sobre a capacidade das áreas verdes urbanas no sequestro e estocagem de carbono.

\section{MATERIAL E MÉTODOS}

\section{1. Área de estudo}

O estudo foi realizado no campus-sede da Universidade Federal de Viçosa (UFV), cuja área total é de 1.359 ha, localizado no Município de Viçosa, Minas Gerais, sob as coordenadas geográficas $42^{\circ} 52^{\prime} \mathrm{W}$ e $20^{\circ}$ 45’S (UFV, 2009).

Percorreram-se todos os estacionamentos, arboretos e vias do campus da UFV, identificando e mensurando todas as árvores com diâmetro do fuste com casca a $1,30 \mathrm{~m}$ de altura do solo (DAP) igual ou superior a $5 \mathrm{~cm}$.

\subsection{Coleta de dados (árvores não palmeiras)}

A partir da caracterização diamétrica das árvores, selecionaram-se 721 árvores-amostra distribuídas em 101 espécies e em diferentes classes de diâmetro, nas quais se mensuraram o DAP com o uso de fita diamétrica e a altura total (Ht) através do hipsômetro Forest Vertex ${ }^{\circledR}$ (Tabela 1).

Posteriormente, para obtenção do volume com casca, cubaram-se rigorosamente os indivíduos arbóreos em pé, através da aplicação sucessiva da expressão de Smalian (HUSCH et al., 2003) até o diâmetro-limite de $5 \mathrm{~cm}$, tanto para o fuste quanto para os galhos. Para isso, utilizou-se o pentaprisma de Wheeler para obtenção dos diâmetros das seções, bem como a baliza graduada para mensuração do comprimento. Quando necessário, escalaram-se os indivíduos arbóreos para facilitar a obtenção das medidas dos galhos mais altos.

\subsection{Coleta de dados (árvores palmeiras)}

No caso das palmeiras presentes no campus, foi realizado o censo (100\% dos indivíduos), sendo todos cubados em pé, do mesmo modo como foi feito para as demais árvores (uso do pentaprisma de Wheeler para

Revista Árvore, Viçosa-MG, v.37, n.6, p.1073-1081, 2013 
Tabela 1 - Número de árvores-amostra por centro de classe de diâmetro e de altura (não palmeiras) utilizadas para gerar equações para estimar o carbono total (CT) e dos galhos (CG) na arborização da Universidade Federal de Viçosa.

Table 1 - Number of sample trees per diameter and height center classes (no palms) used to generate equations to estimate total carbon (TC) and branches carbon (BC) in afforestation at Universidade Federal de Viçosa.

\begin{tabular}{|c|c|c|c|c|c|c|c|c|c|}
\hline & \multicolumn{9}{|c|}{ Centro de classe de altura (m) } \\
\hline & & 2,5 & 7,5 & 12,5 & 17,5 & 22,5 & 27,5 & 32,5 & Total \\
\hline \multirow{28}{*}{ 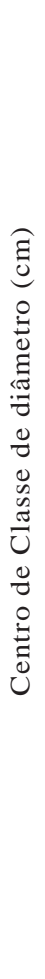 } & 7,5 & 31 & 39 & 10 & 5 & 1 & & & 86 \\
\hline & 12,5 & 18 & 41 & 24 & 6 & 2 & & & 91 \\
\hline & 17,5 & 9 & 40 & 19 & 12 & 3 & 1 & & 84 \\
\hline & 22,5 & 6 & 30 & 25 & 12 & 7 & 2 & & 82 \\
\hline & 27,5 & 2 & 26 & 24 & 8 & 3 & & & 63 \\
\hline & 32,5 & 1 & 21 & 21 & 12 & 5 & 1 & & 61 \\
\hline & 37,5 & 3 & 11 & 25 & 6 & 4 & 2 & & 51 \\
\hline & 42,5 & 1 & 11 & 15 & 4 & 7 & 3 & & 41 \\
\hline & 47,5 & & 13 & 4 & 8 & 4 & 4 & 1 & 34 \\
\hline & 52,5 & & 5 & 5 & 3 & & 1 & 2 & 16 \\
\hline & 57,5 & & 8 & 4 & 2 & 2 & 1 & & 17 \\
\hline & 62,5 & & 3 & 4 & 3 & 2 & 3 & & 15 \\
\hline & 67,5 & & 1 & 3 & 7 & 2 & 1 & & 14 \\
\hline & 72,5 & & 1 & 2 & 4 & 1 & & & 8 \\
\hline & 77,5 & & 3 & 4 & 2 & 2 & 2 & 1 & 14 \\
\hline & 82,5 & & 1 & 3 & 4 & 1 & 1 & & 10 \\
\hline & 87,5 & & 1 & 2 & 1 & & & & 4 \\
\hline & 92,5 & & 1 & 1 & 2 & 2 & & & 6 \\
\hline & 97,5 & & 1 & & 3 & 2 & & & 6 \\
\hline & 102,5 & & 1 & 1 & 1 & & & & 3 \\
\hline & 107,5 & & 1 & 1 & 2 & & & & 4 \\
\hline & 112,5 & & & 1 & & 1 & & & 2 \\
\hline & 117,5 & & & 2 & & 1 & & & 3 \\
\hline & 127,5 & & & 1 & 1 & & & & 2 \\
\hline & 137,5 & & & & 1 & 1 & & & 2 \\
\hline & 157,5 & & & 1 & & & & & 1 \\
\hline & 167,5 & & & 1 & & & & & 1 \\
\hline & Total & 71 & 259 & 203 & 109 & 53 & 22 & 4 & 721 \\
\hline
\end{tabular}

obtenção dos diâmetros das seções e baliza graduada para mensuração do comprimento). No entanto, o volume com casca do estipe de cada indivíduo foi obtido pela expressão de Huber (SOARES et al., 2005). Considerou-se, portanto, o estipe como uma única seção (Tabela 2).

Optou-se pelo método indireto para obtenção do volume, pois não é permitido nem recomendável realizar o abate das árvores presentes em arborização urbana, em especial no campus, dadas a representatividade e importância de tais indivíduos perante as sociedades acadêmica e viçosense.

\subsection{Quantificação de biomassa e carbono}

Quanto às árvores não palmeiras, foram selecionados três indivíduos de cada espécie e de cada centro de classe diamétrica, dos quais se extraiu uma amostra de madeira com casca de cada um com o auxílio de um trado mecânico na porção do DAP, para determinação da densidade básica média da espécie. Como na extração não foi possível a separação da madeira e da casca, analisou-se a densidade de ambos conjuntamente.

Algumas espécies arbóreas cuja tradagem mecânica foi dificultada ou impedida de ser realizada, devido às propriedades de sua madeira, optou-se pela retirada de um disco de madeira de aproximadamente $5 \mathrm{~cm}$ de espessura na altura da medida do DAP e outro na base do galho. No caso das palmeiras, também não foi possível extrair o material com o trado mecânico, pois a amostra se apresentava muito quebradiça e desuniforme em razão das características anatômicas do estipe. Assim, optou-se pela derrubada de um indivíduo por espécie e pela extração de um disco de 5 cm de diâmetro na 
Tabela 2 - Número de árvores-amostra por centro de classe de diâmetro e de altura (palmeiras) utilizadas para gerar equações para estimar o carbono total (CT) na arborização da Universidade Federal de Viçosa.

Table 2 - Number of sample trees per diameter and height center classes (palms) used to generate equations to estimate total carbon (TC) in afforestation at Universidade Federal de Viçosa.

\begin{tabular}{|c|c|c|c|c|c|c|c|c|c|}
\hline & \multicolumn{9}{|c|}{ Centro de classe de altura (m) } \\
\hline & & 2,5 & 7,5 & 12,5 & 17,5 & 22,5 & 27,5 & 32,5 & Total \\
\hline \multirow{20}{*}{ 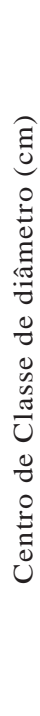 } & 2,5 & 5 & 1 & 3 & & & & & 9 \\
\hline & 7,5 & 40 & 330 & 117 & & & & & 487 \\
\hline & 12,5 & 1 & 67 & 16 & & & & & 84 \\
\hline & 17,5 & & 30 & 5 & 1 & & & & 36 \\
\hline & 22,5 & 1 & 40 & 66 & 40 & 7 & & & 154 \\
\hline & 27,5 & & 16 & 21 & 24 & 14 & & & 75 \\
\hline & 32,5 & & 3 & 7 & 5 & 1 & & & 16 \\
\hline & 37,5 & & 2 & 3 & 3 & 1 & & & 9 \\
\hline & 42,5 & & 4 & 2 & & 1 & & & 7 \\
\hline & 47,5 & & 5 & 1 & 3 & & & & 9 \\
\hline & 52,5 & & & 2 & 1 & & 1 & & 4 \\
\hline & 57,5 & & 2 & 2 & & 1 & & & 5 \\
\hline & 62,5 & & & 3 & 1 & 4 & & & 8 \\
\hline & 67,5 & & & 4 & 3 & 3 & 2 & & 12 \\
\hline & 72,5 & & & 4 & 5 & 3 & 7 & 1 & 20 \\
\hline & 77,5 & & & & 3 & 1 & 9 & 5 & 18 \\
\hline & 82,5 & & & & & & 6 & 2 & 8 \\
\hline & 87,5 & & & & & & 1 & & 1 \\
\hline & 92,5 & & & & & 1 & & & 1 \\
\hline & Total & 47 & 500 & 256 & 89 & 37 & 26 & 8 & 963 \\
\hline
\end{tabular}

altura da medida do DAP, para a quantificação da biomassa e do teor de carbono. A derrubada desses indivíduos foi autorizada pela administração da universidade e, em alguns casos, aproveitou-se do fato de que algumas árvores caíram em razão das fortes chuvas que ocorreram durante o período de pesquisa.

O cálculo da determinação da densidade básica nesses casos foi feito em laboratório, segundo a Norma NBR 11941 (ABNT, 2003), a partir da equação 1.

$$
D b=\frac{m_{3}}{\left(m_{2}-m_{1}\right)}
$$

em que $\mathrm{Db}=$ densidade básica da madeira e da casca, em g. $\mathrm{cm}^{-3} ; \mathrm{m}_{3}=$ massa da amostra seca em estufa a $(105 \pm 2)^{\circ} \mathrm{C}$, em g; $\mathrm{m}_{2}=$ massa do recipiente com água e amostra imersa, em g; e $m_{1}=$ massa do recipiente com água, em g.

A biomassa presente no fuste ou estipe e nos galhos de cada árvore foi obtida pela multiplicação do volume total com casca pela densidade básica da espécie correspondente, conforme a equação 2 .

$$
B_{i}=d_{i}^{*} V_{i}
$$

em que $B_{i}$ = biomassa do fuste ou estipe e de galhos do indivíduo da i-ésima espécie, em kg; $\mathrm{d}_{\mathrm{i}}=$ densidade básica da i-ésima espécie, em kg.m³ $\mathrm{e} \mathrm{V}_{\mathrm{i}}=$ volume mensurado com casca do fuste ou estipe e dos galhos da árvore, pertencente à i-ésima espécie, em m³ .

Para determinação da análise elementar (para quantificação do teor de carbono das amostras), aferiu-se uma massa equivalente a $2,5 \mathrm{mg}( \pm 0,5)$ de serragem absolutamente seca, que foi selecionada em peneiras sobrepostas de 200 e 270 mesh, sendo utilizada a fração retida nesta última. O equipamento utilizado na análise foi o Vario Micro Cube CHNSO. Posteriormente, multiplicou-se o teor de carbono obtido pela biomassa de cada árvore mensurada.

De posse das estimativas de carbono obtidas de cada árvore, bem como das medidas de diâmetro e altura, buscou-se relacionar essas informações por meio de modelos alométricos.

Existem vários modelos alométricos (lineares, não lineares, simples e múltiplos) para estimar o volume, a biomassa e a quantidade de carbono de árvores. Os

Revista Árvore, Viçosa-MG, v.37, n.6, p.1073-1081, 2013 
modelos de Schumacher e Hall (1933) e Spurr (1952) são os mais utilizados, pois resultam em estimativas precisas e sem tendências (AMARO, 2010). Dessa forma, avaliaram-se esses modelos para estimar o carbono total e dos galhos das árvores (equações 3 e 4).

a) Schumacher e Hall (1933)

$$
L n C=\beta_{0}+\beta_{1} * L n D A P+\beta_{2} * L n H t+\varepsilon_{i}
$$

b) Spurr (1952) modificado

$$
\operatorname{Ln} C=\beta_{0}+\beta_{1} * \operatorname{Ln}\left(D A P^{2} * H t\right)+\varepsilon_{i}
$$

em que $\mathrm{Ln}=$ logaritmo neperiano; $\mathrm{C}=$ carbono, em kg; DAP = diâmetro à altura do peito, em cm; $\mathrm{Ht}=$ altura total do indivíduo, em m; $\beta_{0}$ a $\beta_{2}=$ parâmetros do modelo; e $\varepsilon_{\mathrm{i}}=$ erro aleatório.

As estimativas dos parâmetros foram obtidas pelo Método de Mínimos Quadrados Ordinários, com o auxílio do software Statistica 7.0 (STATSOFT, 2009). Além disso, optou-se por utilizar as equações em suas formas lineares, a fim de corrigir eventuais problemas de heterocedasticidade de variância e de normalidade dos erros, conforme Soares e Oliveira (2002).

Sabe-se que a principal perda de biomassa e carbono nas árvores urbanas se dá pela poda de galhos e ramos. Dessa forma, optou-se por testar modelos para estimar o carbono presente em galhos (para árvores não palmeiras), a fim de contribuir para o monitoramento de estudos sobre fixação de carbono na arborização urbana e de emissões de gases de efeito-estufa através das podas.

Na seleção dos melhores modelos para estimativa de carbono, adotaram-se os seguintes critérios, segundo Soares et al. (2005) e Oliveira et al. (2005): a) Análise das medidas de precisão: coeficiente de determinação ajustado ( $\left.\overline{\mathrm{R}}^{2}\right)$ e erro-padrão da estimativa ( $\left.\mathrm{S}_{\mathrm{y} . \mathrm{x}} \%\right)$; b) Análise da significância dos coeficientes das equações pelo teste “t”, de Student; e c) Distribuição gráfica dos resíduos.

\section{RESULTADOS}

\section{1. Árvores (não palmeiras)}

Os valores encontrados para os coeficientes de determinação $\left(\mathrm{R}_{\text {ajust. }}^{2}\right)$ e para os erros-padrão de estimativas $\left(\mathrm{S}_{\text {y.x }}\right.$ \%) das equações ajustadas para estimar o carbono total (CT) e o carbono dos galhos (CG) das árvores presentes no campus-sede da UFV mostraram-se satisfatórios, levando em consideração a grande heterogeneidade dos dados observados.

Entre os modelos avaliados para estimar o carbono total em árvores pertencentes à arborização do campussede da UFV, o de Schumacher e Hall (1933) foi o que se ajustou melhor aos dados e apresentou melhor distribuição dos resíduos em relação ao modelo de Spurr (1952) modificado. Além disso, todos os coeficientes foram estatisticamente significativos, considerando $\mathrm{p}<0,05$ (Tabela 3).

Para estimar o carbono nos galhos, o modelo de Schumacher e Hall (1933) também foi o que se ajustou melhor aos dados e também apresentou melhor distribuição dos resíduos em relação ao modelo de Spurr (1952) modificado (Figura 1). Todos os coeficientes também foram estatisticamente significativos, considerando $\mathrm{p}<0,05$ (Tabela 3).

\subsection{Palmeiras}

Os modelos para estimativa de carbono em palmeiras mostraram-se mais precisos que os modelos estimados para carbono nas demais árvores. Isso se deve à maior uniformidade na forma do fuste, bem como ao fato de não apresentarem bifurcações de galhos. Todos os coeficientes dos modelos foram significativos a 95\% de probabilidade, pelo teste “t”, de Student.

Comparando as medidas de precisão, notou-se que as equações se ajustaram bem aos dados observados, contudo o modelo de Schumacher e Hall (1933) apresentou-se ligeiramente superior (Tabela 4).

Os resíduos do modelo de Schumacher e Hall (1933) ficaram mais homogeneamente distribuídos e com menor amplitude do que o modelo de Spurr (1952) modificado (Figura 1).

\section{DISCUSSÃO}

Notou-se maior concentração de dados nas menores classes de diâmetro (cerca de 56\% dos indivíduos amostrados possuíam até $30 \mathrm{~cm}$ de diâmetro). Além disso, como os indivíduos avaliados são de diferentes espécies e conformações, houve erros maiores nessas classes, como pode ser verificado na Figura 1.

Atualmente, não se encontram equações de biomassa e carbono especificamente desenvolvidas para árvores urbanas no Brasil, e os trabalhos relacionados, em geral,

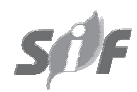

Revista Árvore, Viçosa-MG, v.37, n.6, p.1073-1081, 2013 
Tabela 3 - Estimativas dos parâmetros e medidas de precisão das equações para estimar o carbono total (CT) e dos galhos (CG) na arborização da Universidade Federal de Viçosa.

Table 3 - Parameter estimates and accuracy measures of equations to estimate total (CT) and branches carbon (CG) in urban trees of Universidade Federal de Viçosa.

\begin{tabular}{ccccccc}
\hline \multirow{2}{*}{ Componente } & Modelos & \multicolumn{3}{c}{ Coeficientes } & \multirow{2}{*}{$\begin{array}{c}\mathrm{R}^{2} \text { ajust } \\
(\%)\end{array}$} & $\begin{array}{c}\mathrm{S}_{\mathrm{y} . \mathrm{x}} \\
(\%)\end{array}$ \\
\cline { 3 - 5 } & & $\hat{\beta}_{0}$ & $\hat{\beta}_{0}$ & $\hat{\beta}_{0}$ & & \\
\hline \multirow{2}{*}{ CT } & Schumacher e Hall (1933) & $-0,906586$ & 1,60421 & 0,37162 & 82,67 & 58,88 \\
& Spurr (1952) modificado & $-1,088700$ & 0,70947 & - & 81,42 & 60,97 \\
\multirow{2}{*}{ CG } & Schumacher e Hall (1933) & $-2,052673$ & 1,89903 & 0,24156 & 82,89 & 65,95 \\
& Spurr (1952) modificado & $-2,321309$ & 0,79251 & - & 80,55 & 70,31 \\
\hline
\end{tabular}

Tabela 4 - Estimativas dos parâmetros e medidas de precisão das equações para estimar o carbono das palmeiras do campus-sede da Universidade Federal de Viçosa.

Table 4-Parameter estimates and accuracy measures of equations to estimate carbon in palms located at campus of Universidade Federal de Viçosa.

\begin{tabular}{|c|c|c|c|c|c|}
\hline \multirow{2}{*}{ Modelos } & \multicolumn{3}{|c|}{ Coeficientes } & \multirow{2}{*}{$\begin{array}{c}R^{2} \text { ajust } \\
(\%)\end{array}$} & \multirow{2}{*}{$\begin{array}{l}\mathrm{S}_{\mathrm{y.x}} \\
(\%)\end{array}$} \\
\hline & $\hat{\beta}_{0}$ & $\hat{\beta}_{0}$ & $\hat{\beta}_{0}$ & & \\
\hline Schumacher e Hall (1933) & $-4,46988$ & 1,99082 & 1,06420 & 99,08 & 17,66 \\
\hline Spurr (1952) modificado & $-4,42110$ & 1,00956 & - & 99,07 & 17,75 \\
\hline
\end{tabular}

utilizam equações de florestas nativas para estimar o carbono presente nas árvores das cidades. Entretanto, as árvores urbanas podem apresentar crescimento diferente e diversificado quando comparadas com florestas nativas, pois possuem características peculiares, como maior área útil para desenvolvimento. Podem também receber tratamentos silviculturais diferenciados, ao mesmo tempo que estão mais sujeitas à ação do homem, a estresse e a poluição (NOWAK et al., 2002).

Podem-se observar diferenças quando se comparam os resultados deste trabalho com os encontrados em florestas nativas brasileiras. Amaro (2010), desenvolvendo estudo em Floresta Estacional Semidecidual no Município de Viçosa, obteve uma equação para estimar carbono com coeficiente de determinação ajustado ( $\mathrm{R}^{2}{ }_{\text {ajust. }}$ ) de 95,34\% e erro-padrão de estimativa $\left(\mathrm{S}_{\mathrm{y} \cdot \mathrm{x}}\right)$ de $\pm 23,6 \%$.

Rezende et al. (2006) encontraram valores de $\mathrm{R}^{2}$ ajust, variando entre $98,12 \%$ e $98,64 \%$ e $S_{y . x}$ entre $\pm 25,66$ $\mathrm{e} \pm 40,69 \%$, para modelos de estocagem de carbono em árvores de Cerrado sensu stricto em Brasília.

Miranda et al. (2011), desenvolvendo modelos alométricos para estimativa do carbono total das árvores de diferentes espécies plantadas em áreas de restauração florestal no Estado de São Paulo, obtiveram um $\mathrm{R}^{2}$ acima de $95 \%$ e $S_{y . x}$ de $\pm 31,19 \%$, encontrando resultados ainda melhores quando estratificado de acordo com o ritmo de crescimento das plantas.

Já Scolforo et al. (2008), estudando florestas nativas de Minas Gerais, encontraram $\mathrm{R}^{2}$ ajust. igual a 93,76\% e $_{\text {y.x }}$ de $\pm 39,88 \%$. Entretanto, Johnson e Gerhold (2001) geraram uma equação alométrica para estimar carbono de três espécies arbóreas em duas cidades americanas, encontrando coeficiente de determinação igual a 70,70\%, valor inferior ao obtido neste estudo. Dependendo da escala, das características da espécie ou da população, a variabilidade das estimativas de carbono pode variar entre 60 e 300\% (McHALE et al., 2009), mostrando, desse modo, a importância do aprimoramento das pesquisas na área.

A menor precisão encontrada neste trabalho quando comparada com a de estudos realizados em florestas nativas se deve à variabilidade da arborização do campus-sede da UFV, que possui grande número de espécies de diferentes portes, conformações e idades. Além disso, esses indivíduos recebem diferentes tratos silviculturais, como podas, que interferem no seu desenvolvimento. 


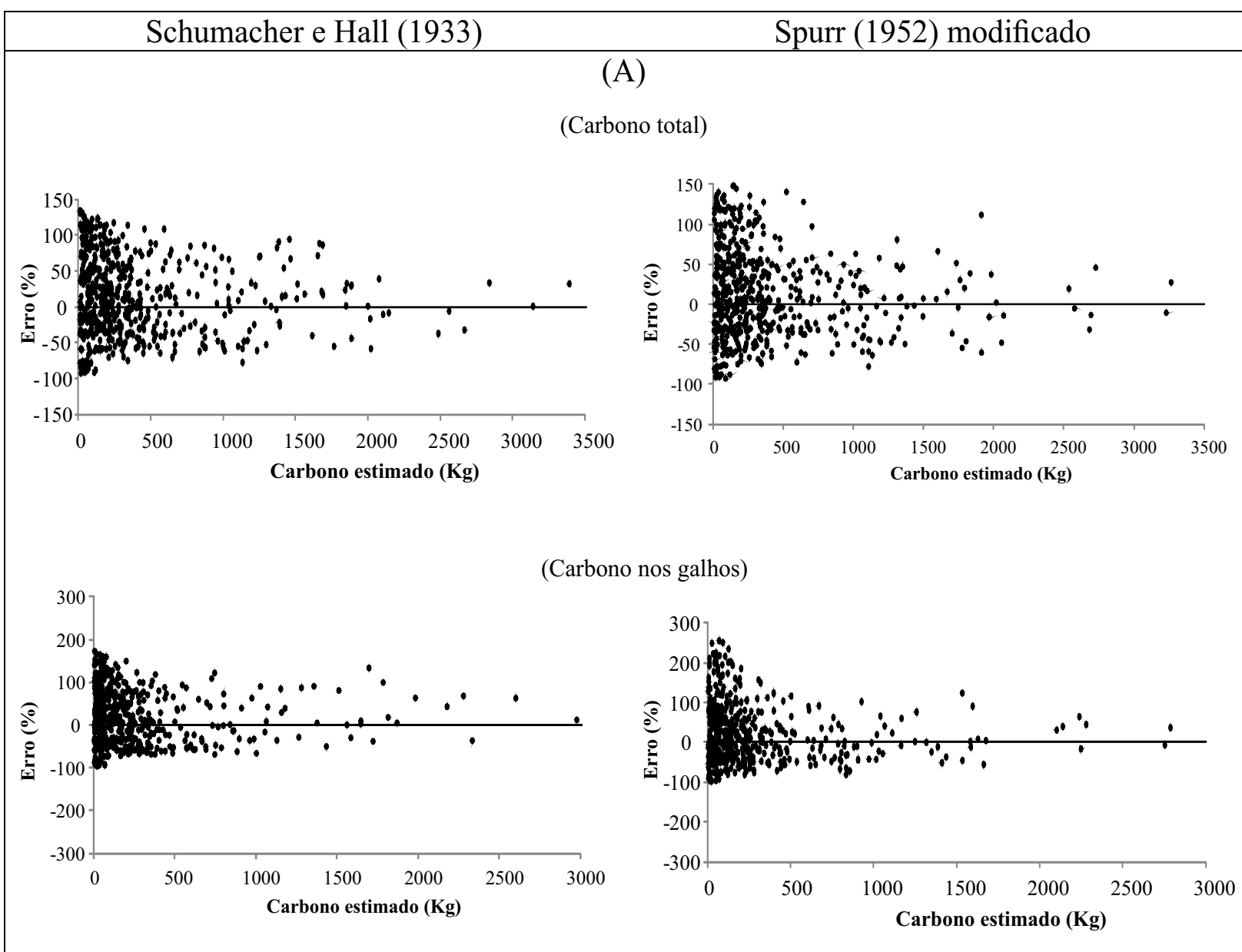

(B)
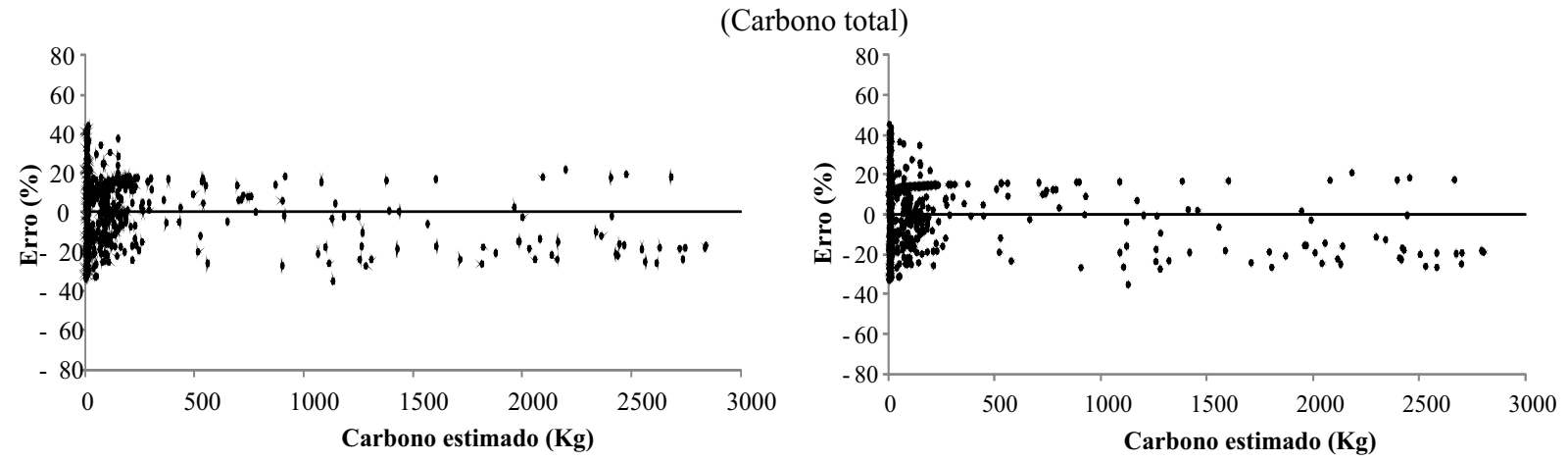

Figura 1 - Distribuição dos resíduos das equações que estimam o carbono total (CT) e o carbono dos galhos (CG) para árvores não palmeiras (A) e carbono total (CT) em palmeiras (B) do campus-sede da Universidade Federal de Viçosa.

Figure 1 - Distribution of the equations residuals that estimate the total carbon (CT) and branches carbon (CG) in no-palms trees (A) and total carbon (CT) in palms (B) located at Universidade Federal de Viçosa 
Maior intensidade de amostragem, principalmente nos indivíduos de maior porte e frequência, poderia melhorar as estimativas, porém, como observado anteriormente, há menor frequência de indivíduos nas maiores classes de diâmetro.

Com relação às palmeiras, notou-se que as medidas de precisão obtidas nos modelos ajustados foram similares às encontradas ao estimar o carbono total em povoamentos homogêneos, como de Eucalyptus spp. (SOARES; OLIVEIRA, 2002; SOARES etal., 2005; GATTO et al., 2011), Acacia mearnsii (SAIDELLES et al., 2009), Araucaria angustifolia e Pinus taeda (SANQUETTA et al., 2003).

Isso foi favorecido em razão da própria conformação anatômica das palmeiras, que se caracterizam por apresentar estipe único e uniforme em toda a sua extensão, não tendo grande variação entre as espécies.

As equações geradas neste estudo podem, portanto, contribuir para a elaboração de projetos de carbono que visem à compensação das emissões de GEE em condições similares ou dentro do próprio município, facilitando a obtenção de resultados mais precisos. Ademais, essas equações podem incentivar outros estudos de arborização urbana, principalmente vinculados à área de carbono.

\section{CONCLUSÕES}

Pode-se concluir que as equações referentes ao modelo de Schumacher e Hall (1933) foram as que tiveram melhor ajuste aos dados observados de carbono total e de galhos nas árvores não palmeiras e de carbono total nas palmeiras. Nas árvores não palmeiras do campus-sede da Universidade Federal de Viçosa, o carbono total e o carbono dos galhos podem ser estimados em kg, em razão do Diâmetro àAltura do Peito (DAP, em cm) e da Altura Total (Ht, em m), por $-0,906586+1,60421 * \mathrm{LnDAP}+0,37162 * \mathrm{LnHt}$ e por $-2,052673+1,89903 * \mathrm{LnDAP}+0,24156 * \mathrm{LnHt}$, respectivamente. Nas palmeiras, o carbono total pode ser estimadopor -4,46988+1,99082*LnDAP+1,06420*LnHt.

\section{AGRADECIMENTOS}

Ao Conselho Nacional de Desenvolvimento Científico e Tecnológico (CNPq) e à Fundação de Amparo à Pesquisa do Estado de Minas Gerais (FAPEMIG), pelo apoio financeiro e pela concessão de bolsa.

\section{REFERÊNCIAS}

ASSOCIAÇÃO BRASILEIRA DE NORMAS TÉCNICAS - ABNT. NBR 11941: Madeira Determinação da densidade básica. Rio de Janeiro: 2003. 6p.

AMARO, M. A. Quantificação do estoque volumétrico, de biomassa e de carbono em uma Floresta Estacional Semidecidual no município de Viçosa, MG. 2010. 168f. Tese (Doutorado em Ciência Florestal) Universidade Federal de Viçosa, Viçosa, MG, 2010.

GATTO, A. et al. Estoque de carbono na biomassa de plantações de eucalipto na região centro-leste do estado de Minas Gerais.

Revista Árvore, v.35, n.4, p.895-905, 2011.

HUSCH, B.; BEERS, T. W.; KERSHAW JUNIOR, J. A. Forest mensuration. 4.ed. Malabar: Krieger Publishing Company, 2003. 443p.

JOHNSON, A. D.; GERHOLD, H. D. Carbon storage by utility-compatible trees. Journal of Arboriculture, v.27, n.2, p.57-68, 2001.

KETTERINGS, Q. M. et al. Reducing uncertainty in the use of allometric biomass equations for predicting aboveground tree biomass in mixed secondary forests. Forest Ecology and Management, v.146, n.1/3, p.199-209, 2001.

MCHALE, M. R. et al. Urban Forest biomass estimates: is it important to use allometric relationships developed specifically for urban trees? Urban Ecosyst, v.12, n.1, p.95-113, 2009.

MIRANDA, D. L. C.; MELO, A. C. G.; SANQUETTA, C. R. Equações alométricas para estimativa de biomassa e carbono em árvores de reflorestamentos de restauração. Revista Árvore, v.35, n.3, Edição Especial, p.679-689, 2011.

NOWAK, D. J. et al. Brooklyn's urban forest. GTR NE-290. Newtown Square: USDA Forest Service, Northeastern Research Station, 2002. 112p.

Revista Árvore, Viçosa-MG, v.37, n.6, p.1073-1081, 2013 
OLIVEIRA, M. L. R. et al. Equações de volume de povoamento para fragmentos florestais naturais no município de Viçosa, Minas Gerais. Revista Árvore, v.29, n.2, p.213-225, 2005.

PAIXÃO, F. A. et al. Quantificação do estoque de carbono e avaliação econômica de diferentes alternativas de manejo em um plantio de eucalipto. Revista Árvore, v.30, n.3, p.411-420, 2006.

REZENDE, A. V. et al. Comparação de modelos matemáticos para estimativa do volume, biomassa e estoque de carbono da vegetação lenhosa de um cerrado sensu stricto em Brasília, DF.

Scientia Forestalis, n.71, p.65-76, 2006.

RIBEIRO, S. C. et al. Above-and belowground biomass in a Brazilian Cerrado. Forest Ecology and Management, v.262, n.3, p.491-499, 2011.

SAIDELLES, F. L. F. et al. Uso de equações para estimar carbono orgânico em plantações de Acacia mearnsii de Wild. No Rio Grande do Sul - Brasil. Revista Árvore, v.33, n.5, p.907-915, 2009.

SAMPSON, R. N.; MOLL, G. A.; KIELBASO, J. Opportunities to increase urban forests and the potential impacts on carbon storage and conservation. In: HAIR, D.; SAMPSON, R. N. (Ed.) Forests and global change. Opportunities for increasing forest cover american forests. Washington: 1992. v.1. p.51-72.

SANQUETTA, C. R. et al. Relações individuais de biomassa e conteúdo de carbono em plantações de Araucaria angustifolia e Pinus taeda no sul do estado do Paraná, Brasil. Revista

Acadêmica: Ciências Agrárias e

Ambientais, v.1, n.3, p.33-40, 2003.
SCHUMACHER, F. X.; HALL, F. D. S.

Logarithmic expression of timber-tree volume. Journal Agricultural Research, v.47, n.9, p.719-734, 1933.

SCOLFORO, J. R. S.; OLIVEIRA, A. D.; ACERBI JÚNIOR, F. W. (Ed.). Inventário florestal de Minas Gerais: equações de volume, peso de matéria seca e carbono para diferentes fitofisinomias da flora nativa. Lavras: Universidade Federal de Lavras, 2008. 216p.

SOARES, C. P. B.; LEITE, H. G.; GÖRGENS, E. B. Equações para estimar o estoque de carbono no fuste de árvores individuais e em plantios comerciais de eucalipto. Revista Árvore, v.29, n.5, p.711-718, 2005.

SOARES, C. P. B.; OLIVEIRA, M. L. R. Equações para estimar a quantidade de carbono na parte aérea de árvores de eucalipto em Viçosa, Minas Gerais. Revista Árvore, v.26, n.5, p.533-539, 2002.

SPURR, S. H. Forest inventory. New York: The Ronald Press Company, 1952. 476p.

STATSOFT, Inc. STATISTICA (data analysis software system). version 8 . Cary: 2009.

UNIVERSIDADE FEDERAL DE VIÇOSA - UFV. UFV em números (2009). Disponível em: <http://www.ufv.br/proplan/ufvnumeros/ numeros2009.pdf>. Acesso em: 10 set. 2012.

URBANO, E. et al. Equações para estimar o peso de carbono fixado em árvores de Mimosa scabrella Bentham (Bracatinga) em povoamentos nativos. Cerne, v.14, n.3, p.194-203, 2008. 
